\title{
NULL CONTROLLABILITY OF NEUTRAL EVOLUTION INTEGRODIFFERENTIAL SYSTEMS WITH INFINITE DELAY
}

\author{
K. BALACHANDRAN AND A. LEELAMANI
}

Received 29 August 2004; Revised 30 May 2005; Accepted 26 March 2006

We establish a set of sufficient conditions for the controllability of nonlinear neutral evolution integrodifferential systems with infinite delay in Banach spaces. The results are established by using the Sadovskil fixed point theorem and generalize the previous results.

Copyright (c) 2006 K. Balachandran and A. Leelamani. This is an open access article distributed under the Creative Commons Attribution License, which permits unrestricted use, distribution, and reproduction in any medium, provided the original work is properly cited.

\section{Introduction}

The concept of controllability plays a major role in finite-dimensional control theory so it is natural to try to generalize this to infinite dimensions. Controllability is the property of being able to steer between two arbitrary points in the state space. For continuous time-invariant linear systems in finite-dimensional space the concepts of controllability and reachability are equivalent, and they are independent of the time. But in infinitedimensional space, the situation is more complex, and many different types of controllability and reachability have been studied in the literature. A weaker condition than exact controllability is the property of being able to steer all points exactly to the origin. This has important connections with the concept of stabilizability. Several authors have studied the null controllability of various kinds of dynamical systems [4, 5].

Neutral differential equations arise in many areas of applied mathematics and such equations have received much attention in recent years. The theory of functional differential equation with unbounded delay has been studied by several authors [6]. Almost all the work deals with the Cauchy problem

$$
x^{\prime}(t)=F\left(t, x_{t}\right), \quad t \geq \sigma, \quad x_{\sigma}=\varphi,
$$

where $x_{t}$ represents the "history" of $x$ at $t$, the values $x(t)$ belong to some finite-dimensional space, and $F$ is a function, usually continuous on appropriate spaces. Nevertheless, 
this class of equations does not include partial integrodifferential equations with infinite delay, which arise, for example, in the study of heat conduction in materials with memory or population dynamics for spatially distributed populations. Besides, it is well known that the behavior of the first- and second-order abstract Cauchy problem is different in many aspects. For these reasons, there has been an increasing interest in studying equations that can be described in the form

$$
x^{\prime}(t)=A x(t)+F\left(t, x_{t}\right), \quad t \geq \sigma,
$$

where $A$ is the infinitesimal generator of a strongly continuous semigroup of linear operators on a Banach space $X$. We call these equations as abstract retarded functional differential equations.

Similarly there exists an extensive theory for ordinary neutral functional differential equations, which includes qualitative behavior of classes of such equations and applications to biological and engineering processes. However, for partial neutral functional differential equations very little is known [14]. Our purpose here is to study the controllability of equations that can be modelled in the form

$$
\frac{d}{d t}\left(x(t)+F\left(t, x_{t}\right)\right)=A x(t)+G\left(t, x_{t}\right), \quad t \geq \sigma
$$

where the initial conditions $x_{\sigma}$ and $F$ and $G$ are appropriate functions. These equations will be called abstract neutral functional differential equations with unbounded delay. As a motivation example for this class of equations we consider the boundary value problem

$$
\begin{gathered}
\frac{\partial}{\partial t}\left[u(t, \xi)+\int_{-\infty}^{t} \int_{0}^{\pi} b(s-t, \eta, \xi) u(s, \eta) d \eta d s\right] \\
=\frac{\partial^{2}}{\partial \xi} u(t, \xi)+a_{0}(\xi) u(t, \xi)+a_{1}(t, \xi)+\int_{-\infty}^{t} a(s-t) u(s, \xi) d s, \quad t \geq 0,0 \leq \xi \leq \pi \\
u(t, 0)=u(t, \pi)=0, \quad t \geq 0 \\
u(\theta, \xi)=\phi(\theta, \xi), \quad \theta \leq 0,0 \leq \xi \leq \pi,
\end{gathered}
$$

where the functions $a_{0}, a, a_{1}, b$, and $\phi$ satisfy appropriate conditions.

These problems arise from control systems described by abstract retarded functional differential equations with a feedback control governed by a proportional integrodifferential law $[1,16]$. On the other hand, some abstract retarded functional differential equations can be conveniently transformed into abstract neutral functional differential equations. Consider (1.2) with

$$
F(\varphi)=\int_{-\infty}^{0} C(-\theta) \varphi(\theta) d \theta
$$

where $C$ is a strongly continuous map of continuous operators from $X$ into $X$. Assume that we can decompose $C(s)=L(s)+N(s)$ where $L$ and $N$ are also strongly continuous 
maps of continuous operators and further the $L(s)$ are linear. We define the operator $V(t)$ by

$$
V(t) x=\int_{0}^{t} L(s) x d s
$$

then (1.2) can be transformed into an abstract neutral functional differential equation

$$
\frac{d}{d t}\left[x(t)+\int_{-\infty}^{t} V(t-s) x(s) d s\right]=A x(t)+\int_{-\infty}^{t} N(t-s) x(s) d s,
$$

which has the form (1.3) and in some cases, depending on $V$ and $N$, it is easier to treat than the original equation. Motivation for neutral functional differential equations can be found in $[3,5,12-14]$.

There are several papers which have appeared on the controllability of nonlinear systems in infinite-dimensional spaces [4]. Balachandran and Anandhi [2] discussed the controllability of neutral functional integrodifferential systems in abstract phase space with the help of Schauder's fixed point theorem. Recently Fu [8, 9] studied the same problem in abstract phase space for neutral functional differential systems and nonlinear neutral systems with unbounded delay by utilizing the Sadovskii fixed point theorem. In this paper we will study the null controllability of neutral evolution integrodifferential system with infinite delay by utilizing the technique of Fu [9]. The results are a generalization of the results established by $\mathrm{Fu}[8,9]$.

Consider the neutral functional integrodifferential system of the form

$$
\begin{gathered}
\frac{d}{d t}\left[x(t)-g\left(t, x_{t}, \int_{0}^{t} h\left(t, s, x_{s}\right) d s\right)\right]=-A(t) x(t)+B u(t)+f\left(t, x_{t}, \int_{0}^{t} q\left(t, s, x_{s}\right) d s\right), \quad t \geq 0, \\
x_{0}=\phi \in \mathscr{B},
\end{gathered}
$$

where the state variable $x(\cdot)$ takes values in the Banach space $X$, and the control function $u(\cdot)$ is in $L^{2}(J, U)$, a Banach space of admissible control functions with $U$ as a Banach space and $J=[0, a]$ and $B$ is a bounded linear operator from $U$ into $X$. The unbounded linear operator $-A(t)$ generates an analytic semigroup and $h: J \times J \times \mathscr{B} \rightarrow X, q: J \times J \times$ $\mathscr{B} \rightarrow X, f, g: J \times \mathscr{B} \times X \rightarrow X$ are appropriate functions and $\mathscr{B}$ is the phase space to be specified later.

\section{Preliminaries}

Throughout this paper $X$ will be a Banach space with norm $\|\cdot\|$. For the family $\{A(t)$ : $0 \leq t \leq a\}$ of linear operators, we assume the following hypotheses.

(B1) The domain $D(A)$ of $\{A(t): 0 \leq t \leq a\}$ is dense in the Banach space $X$ and independent of $t . A(t)$ is a closed linear operator.

(B2) For each $t \in[0, a]$, the resolvent $R(\lambda, A(t))=(\lambda I-A(t))^{-1}$ of $A(t)$ exists for all $\lambda$ with $\operatorname{Re} \lambda \leq 0$ and $\|R(\lambda, A(t))\| \leq C(|\lambda|+1)^{-1}$. 
4 Null controllability of neutral integrodifferential systems

(B3) For any $t, s, \tau \in[0, a]$, there exists a $0<\delta<1$ and $K>0$ so that

$$
\left\|(A(t)-A(\tau)) A^{-1}(s)\right\| \leq K|t-\tau|^{\delta} .
$$

(B4) For each $t \in[0, a]$ and some $\lambda \in \rho(A(t))$, the resolvent set of $A(t), R(\lambda, A(t))$ is a compact operator.

Then the family $\{A(t): 0 \leq t \leq a\}$ generates a unique linear evolution system $\{U(t, s)$ : $0 \leq s \leq t \leq a\}$ satisfying the following properties.

(a) $U(t, s) \in L(X)$ the space of bounded linear transformation on $X$, whenever $0 \leq$ $s \leq t \leq a$ and for each $x \in X$, the mapping $(t, s) \rightarrow U(t, s) x$ is continuous.

(b) $U(t, s) U(s, \tau)=U(t, \tau)$ for $0 \leq \tau \leq s \leq t \leq a$.

(c) $U(t, t)=I$.

(d) $U(t, s)$ is a compact operator whenever $t, s>0$.

Condition (B4) ensures that the generated evolution system satisfies (d). In fact, let $\exp (-\tau A(t))$ denote the analytic semigroup having infinitesimal generator $A(t)$, we obtain the estimation $\|\exp (-\tau A(t))\| \leq K$. Friedman [7] constructed a family of bounded linear operators $\{\Phi(t, \tau): 0 \leq \tau \leq t \leq a\}$ with $\|\Phi(t, \tau)\| \leq K|t-\tau|^{\alpha-1}$ that provide the following representation for $U(t, s)$ :

$$
U(t, s)=e^{-(t-s) A(s)}+\int_{s}^{t} e^{-(t-\tau) A(\tau)} \Phi(\tau, s) d \tau
$$

We choose a sequence $\left\{\epsilon_{n}\right\}$ which decreases to zero. If $\epsilon_{n}<t-s$, we define

$$
U_{n}(t, s)=e^{-(t-s) A(s)}+\int_{s}^{t-\epsilon_{n}} e^{-(t-\tau) A(\tau)} \Phi(\tau, s) d \tau .
$$

The condition (B4) implies that $\exp (-\tau A(t))$ is compact whenever $\tau>0$, then each $U_{n}(t, s)$ is compact and we have

$$
\left\|U(t, s)-U_{n}(t, s)\right\| \leq \epsilon_{n} K^{2}\left|t-\epsilon_{n}-s\right|^{\alpha-1}
$$

Thus $U(t, s)$ is the limit of a sequence of compact operators in the uniform operator topology, which implies that $(\mathrm{d})$ is true.

For the evolution system $\{U(t, s): 0 \leq s \leq t \leq a\}$, the following properties are well known [18]:

(i) there is a constant $M_{1}>0$ such that $\|U(t, s)\| \leq M_{1}$ for all $0 \leq s \leq t \leq a$,

(ii) there is a constant $M_{2}>0$ such that $\left\|A^{-1}(t)\right\| \leq M_{2}$ for $0 \leq t \leq a$,

(iii) the family of operators $\{U(t, s), t>s\}$ is continuous in $t$ in the uniform operator topology uniformly for $s$.

We need the following fixed point theorem due to Sadovskii [17].

Theorem 2.1 (Sadovskiǔ). Let $P$ be a condensing operator on a Banach space $X$, that is, $P$ is continuous and takes bounded sets into bounded sets, and let $\alpha(P(B)) \leq \alpha(B)$ for every bounded set $B$ of $X$ with $\alpha(B)>0$. If $P(H) \subset H$ for a convex, closed, and bounded set $H$ of $X$, then $P$ has a fixed point in $H$ (where $\alpha(\cdot)$ denotes Kuratowski's measure of noncompactness). 
We assume that the delay $x_{t}:(-\infty, 0] \rightarrow X$ defined by $x_{t}(\theta)=x(t+\theta)$ belongs to some abstract phase space $\mathscr{B}$, which will be a linear space of functions mapping $(-\infty, 0]$ into $X$ endowed with the seminorm $\|\cdot\|_{\circledast}$ and satisfying the following axiom $[10,11,15]$.

(A1) If $x:(-\infty, a) \rightarrow X, a>0$, is continuous on $[0, a]$ and $x_{0} \in \mathscr{B}$, then for every $t \in[0, a)$ the following conditions hold:

(i) $x_{t}$ is in $\mathscr{B}$,

(ii) $\|x(t)\| \leq H\left\|x_{t}\right\|_{\Re}$,

(iii) $\left\|x_{t}\right\|_{\mathscr{B}} \leq K(t) \sup \{\|x(s)\|: 0 \leq s \leq t\}+M(t)\left\|x_{0}\right\|_{\mathscr{B}}$.

Here $H \geq 0$ is a constant, $K, M:[0, \infty) \rightarrow[0, \infty), K$ is continuous and $M$ is locally bounded, and $H, K, M$ are independent of $x(t)$.

(A2) For the function $x(\cdot)$ in $(\mathrm{A} 1), x_{t}$ is a $\mathscr{B}$-valued continuous function on $[0, a]$.

(A3) The space $\mathscr{B}$ is complete.

We assume the following assumptions on the system (1.8).

(H1) $g: J \times \mathscr{B} \times X \rightarrow X$ is a continuous function, and there exist constants $L, L_{1}>0$ such that the function $A(t) g$ satisfies the Lipschitz condition

$$
\left\|A(t) g\left(s_{1}, \phi_{1}, \eta_{1}\right)-A(t) g\left(s_{2}, \phi_{2}, \eta_{2}\right)\right\| \leq L\left[\left|s_{1}-s_{2}\right|+\left\|\phi_{1}-\phi_{2}\right\|_{\mathscr{B}}+\left\|\eta_{1}-\eta_{2}\right\|_{X}\right]
$$

for $0 \leq s_{1}, s_{2} \leq a, \phi_{1}, \phi_{2} \in \mathscr{B}, \eta_{1}, \eta_{2} \in X$, and the inequality

$$
\|A(t) g(t, \phi, \eta)\| \leq L_{1}\left[\|\phi\|_{\Re}+\|\eta\|_{X}+1\right]
$$

holds for $t \in[0, a], \phi \in \mathscr{B}, \eta \in X$.

(H2) $h: J \times J \times \mathscr{B} \rightarrow X$ is continuous and there exist constants $L_{2}, L_{3}>0$ such that the function $A(t) h$ satisfies the Lipschitz condition

$$
\left\|A(t) h\left(t_{1}, s, \psi_{1}\right)-A(t) h\left(t_{2}, s, \psi_{2}\right)\right\| \leq L_{2}\left[\left|t_{1}-t_{2}\right|+\left\|\psi_{1}-\psi_{2}\right\|_{\mathscr{B}}\right]
$$

for $0 \leq t_{1}, t_{2} \leq a, \psi_{1}, \psi_{2} \in \mathscr{B}$, and the inequality

$$
\|A(t) h(t, s, \psi)\| \leq L_{3}\left[\|\psi\|_{\mathscr{B}}+1\right]
$$

holds for $t, s \in[0, a], \psi \in \mathscr{B}$.

(H3) The function $q: J \times J \times \mathscr{B} \rightarrow X$ is a continuous function such that there is a positive function $a \in L^{1}([0, a])$ such that

$$
\left|q\left(t, s, x_{s}\right)\right| \leq a(t, s)|| x_{s} \|, \quad \delta=\sup _{t \in J} \int_{0}^{t} a(t, s) d s .
$$

(H4) The function $f: J \times \mathscr{B} \times X \rightarrow X$ satisfies the following conditions.

(i) For each $t \in[0, a]$, the function $f(t, \cdot, \cdot): \mathscr{B} \times X \rightarrow X$ is continuous and for each $(\phi, x) \in \mathscr{B} \times X$, the function $f(\cdot, \phi, x): J \rightarrow X$ is strongly measurable.

(ii) For each positive number $k$, there is a positive function $\mu_{k} \in L^{1}([0, a])$ such that

$$
\sup _{\|x\|,\|y\| \leq k}\|f(t, x, y)\| \leq \mu_{k}(t), \quad \lim \inf _{k \rightarrow \infty} \frac{1}{k} \int_{0}^{a} \mu_{k}(s) d s=\gamma<\infty .
$$


6 Null controllability of neutral integrodifferential systems

(H5) The linear operator $W$ from $L^{2}(J ; U)$ into $X$ is defined by

$$
W u=\int_{0}^{a} U(a, s) B u(s) d s
$$

which induces an invertible operator $\widetilde{W}$ defined on $L^{2}(J ; U) / \operatorname{ker} W$, and there exists a constant $M_{3}>0$ such that $\left\|B \widetilde{W}^{-1}\right\| \leq M_{3}$.

The system (1.8) is said to be (local) null controllable on the interval $[0, a]$ if for every initial function $\phi \in(\Omega \subset) \mathscr{B}$ there exists a control $u \in L^{2}([0, a] ; U)$ such that the mild solution $x(\cdot)$ of $(1.8)$ satisfies $x(a)=0$.

For simplicity let us take $K_{a}=\sup \{K(t), 0 \leq t \leq a\}$ :

$$
\begin{gathered}
L^{*}=\left(M_{2} L K_{a}+a M_{1} L K_{a}\right)\left(1+a M_{2} L_{2}\right), \\
M^{*}=\left(1+a M_{1} M_{3}\right)(N+1)\left(1+a M_{2} L_{3}\right)\left[L_{1}\left(M_{2}+a M_{1}\right)\right] \\
+a M_{1} M_{3}\left[M_{1}(\|\phi(0)\|+\|g(0, \phi, 0)\|)\right]+M_{1}\|g(0, \phi, 0)\| .
\end{gathered}
$$

(H6) Further assume that

$$
\begin{gathered}
L^{*}<1, \\
\left(1+a M_{1} M_{3}\right)\left[\left(M_{2} L_{1} K_{a}+a M_{1} L_{1} K_{a}\right)\left(1+a M_{2} L_{3}\right)+M K_{a}(1+\delta) \gamma\right] \leq 1 .
\end{gathered}
$$

\section{Main results}

THeOREM 3.1. If the assumptions (H1)-(H6) are satisfied and $\phi \in \mathscr{B}$, then the system (1.8) is null controllable on the interval $[0, a]$.

Proof. Using the assumption (H5), for an arbitrary function $x(\cdot)$ define the control

$$
\begin{aligned}
u(t)=\widetilde{W}^{-1}\{ & -U(a, 0)[\phi(0)-g(0, \phi, 0)]-g\left(a, x_{a}, \int_{0}^{a} h\left(a, s, x_{s}\right) d s\right) \\
& -\int_{0}^{a} U(a, s) A(s) g\left(s, x_{s}, \int_{0}^{s} h\left(s, \tau, x_{\tau}\right) d \tau\right) d s \\
& \left.-\int_{0}^{a} U(a, s) f\left(s, x_{s}, \int_{0}^{s} q\left(s, \tau, x_{\tau}\right) d \tau\right) d s\right\}(t) .
\end{aligned}
$$

It will be shown that when using this control the operator $S$ defined by

$$
\begin{aligned}
(S x)(t)= & U(t, 0)[\phi(0)-g(0, \phi, 0)]+g\left(t, x_{t}, \int_{0}^{t} h\left(t, s, x_{s}\right) d s\right) \\
& +\int_{0}^{t} U(t, s) A(s) g\left(s, x_{s}, \int_{0}^{s} h\left(s, \tau, x_{\tau}\right) d \tau\right) d s \\
& +\int_{0}^{t} U(t, s)\left[B u(s)+f\left(s, x_{s}, \int_{0}^{s} q\left(s, \tau, x_{\tau}\right) d \tau\right)\right] d s, \quad 0 \leq t \leq a,
\end{aligned}
$$


has a fixed point $x(\cdot)$. Then $x(\cdot)$ is a mild solution of system $(1.8)$, and it is easy to verify that

$$
x(a)=(S x)(a)=0
$$

which implies that the system is null controllable.

Next we will prove that the operator $S$ has a fixed point.

Let $y(\cdot):(-\infty, a) \rightarrow X$ be the function defined by

$$
y(t)= \begin{cases}U(t, 0) \phi(0), & t \geq 0, \\ \phi(t), & -\infty<t<0,\end{cases}
$$

then $y_{0}=\phi$ and the map $t \rightarrow y_{t}$ is continuous. Take $N=\sup \left\{\left\|y_{t}\right\|_{\overparen{B}}: 0 \leq t \leq a\right\}$. For each $z \in C([0, a] ; X), z(0)=0$, we denote by $\bar{z}$ the function defined by

$$
\bar{z}(t)= \begin{cases}z(t), & 0 \leq t \leq a, \\ 0, & -\infty<t<0 .\end{cases}
$$

If $x(\cdot)$ satisfies the mild solution of (1.8), we can decompose it as $x(t)=z(t)+y(t), 0 \leq$ $t \leq a$, which implies that $x_{t}=\bar{z}_{t}+y_{t}$ for every $0 \leq t \leq a$, and the function $z(\cdot)$ satisfies

$$
\begin{aligned}
z(t)= & -U(t, 0) g(0, \phi, 0)+g\left(t, \bar{z}_{t}+y_{t}, \int_{0}^{t} h\left(t, s, \overline{z_{s}}+y_{s}\right) d s\right) \\
& +\int_{0}^{t} U(t, s) A(s) g\left(s, \bar{z}_{s}+y_{s}, \int_{0}^{s} h\left(s, \tau, \overline{z_{\tau}}+y_{\tau}\right) d \tau\right) d s \\
& +\int_{0}^{t} U(t, s) B u(s) d s+\int_{0}^{t} U(t, s) f\left(s, \bar{z}_{s}+y_{s}, \int_{0}^{s} q\left(s, \tau, \overline{z_{\tau}}+y_{\tau}\right) d \tau\right) d s .
\end{aligned}
$$

Let $P$ be the operator on $C([0, a] ; X)$ defined by

$$
\begin{aligned}
(P z)(t)= & -U(t, 0) g(0, \phi, 0)+g\left(t, \bar{z}_{t}+y_{t}, \int_{0}^{t} h\left(t, s, \bar{z}_{s}+y_{s}\right) d s\right) \\
& +\int_{0}^{t} U(t, s) A(s) g\left(s, \bar{z}_{s}+y_{s}, \int_{0}^{s} h\left(s, \tau, \overline{z_{\tau}}+y_{\tau}\right) d \tau\right) d s \\
& +\int_{0}^{t} U(t, s) B u(s) d s+\int_{0}^{t} U(t, s) f\left(s, \bar{z}_{s}+y_{s}, \int_{0}^{s} q\left(s, \tau, \overline{z_{\tau}}+y_{\tau}\right) d \tau\right) d s .
\end{aligned}
$$

Obviously the operator $S$ has a fixed point if and only if $P$ has a fixed point, so we have to prove that $P$ has a fixed point.

For each positive number $k$, let

$$
B_{k}=\{z \in C([0, a] ; X): z(0)=0,\|z(t)\| \leq k, 0 \leq t \leq a\}
$$


then $B_{k}$, for each $k$, is a bounded, closed, convex set in $C([0, a] ; X)$. Since by $(2.6)$ the following relation holds:

$$
\left\|U(t, s) A(s) g\left(s, \bar{z}_{s}+y_{s}, \int_{0}^{s} h\left(s, \tau, \bar{z}_{\tau}+y_{\tau}\right) d \tau\right)\right\| \leq M L_{1}\left(1+a M_{2} L_{3}\right)\left(k K_{a}+N+1\right),
$$

then it follows that $U(t, s) A(s) g\left(s, \bar{z}_{s}+y_{s}, \int_{0}^{s} h\left(s, \tau, \overline{z_{\tau}}+y_{\tau}\right) d \tau\right)$ is integrable on $[0, a]$, so $P$ is well defined on $B_{k}$. We claim that there exists a positive number $k$ such that $P B_{k} \subseteq B_{k}$. If it is not true, then for each positive number $k$, there is a function $z_{k} \in B_{k}$ but $P z_{k} \notin B_{k}$, that is, $\left\|P z_{k}(t)\right\|>k$ for some $t \in[0, a]$. However, on the other hand, we have

$$
\begin{aligned}
& k \leq\left\|\left(P z_{k}\right)(t)\right\| \\
& =\|-U(t, 0) g(0, \phi, 0)+g\left(t, \bar{z}_{k, t}+y_{t}, \int_{0}^{t} h\left(t, s, \bar{z}_{k, s}+y_{s}\right) d s\right) \\
& +\int_{0}^{t} U(t, s) A(s) g\left(s, \bar{z}_{k, s}+y_{s}, \int_{0}^{s} h\left(s, \tau, \bar{z}_{k, \tau}+y_{\tau}\right) d \tau\right) d s \\
& +\int_{0}^{t} U(t, s) B \widetilde{W}^{-1}\{-U(a, 0)[\phi(0)-g(0, \phi, 0)] \\
& -g\left(a, \bar{z}_{k, a}+y_{a}, \int_{0}^{a} h\left(a, s, \bar{z}_{k, s}+y_{s}\right) d s\right) \\
& -\int_{0}^{a} U(a, \tau) A(\tau) g\left(\tau, \bar{z}_{k, \tau}+y_{\tau}, \int_{0}^{\tau} h\left(\tau, \eta, \bar{z}_{k, \eta}+y_{\eta}\right) d \eta\right) d \tau \\
& \left.-\int_{0}^{a} U(a, \tau) f\left(\tau, \bar{z}_{k, \tau}+y_{\tau}, \int_{0}^{\tau} q\left(\tau, \eta, \bar{z}_{k, \eta}+y_{\eta}\right) d \eta\right) d \tau\right\}(s) d s \\
& +\int_{0}^{t} U(t, s) f\left(s, \bar{z}_{k, s}+y_{s}, \int_{0}^{s} q\left(s, \tau, \bar{z}_{k, \tau}+y_{\tau}\right) d \tau\right) d s \| \\
& \leq M_{1}\|g(0, \phi, 0)\|+\left\|g\left(t, \bar{z}_{k, t}+y_{t}, \int_{0}^{t} h\left(t, s, \bar{z}_{k, s}+y_{s}\right) d s\right)\right\| \\
& +\int_{0}^{t}\left\|U(t, s) A(s) g\left(s, \bar{z}_{k, s}+y_{s}, \int_{0}^{s} h\left(s, \tau, \bar{z}_{k, \tau}+y_{\tau}\right) d \tau\right)\right\| d s \\
& +\int_{0}^{t} M_{1} M_{3}\left\{M_{1}[\|\phi(0)\|+\|g(0, \phi, 0)\|]+\left\|g\left(a, \bar{z}_{k, a}+y_{a}, \int_{0}^{a} h\left(a, s, \bar{z}_{k, s}+y_{s}\right) d s\right)\right\|\right. \\
& +\int_{0}^{a}\left\|U(a, \tau) A(\tau) g\left(\tau, \bar{z}_{k, \tau}+y_{\tau}, \int_{0}^{\tau} h\left(\tau, \eta, \bar{z}_{k, \eta}+y_{\eta}\right) d \eta\right) d \tau\right\| \\
& \left.+M_{1} \int_{0}^{a}\left\|f\left(\tau, \bar{z}_{k, \tau}+y_{\tau}, \int_{0}^{\tau} q\left(\tau, \eta, \bar{z}_{k, \eta}+y_{\eta}\right) d \eta\right) d \tau\right\|\right\}(s) d s \\
& +M_{1} \int_{0}^{t}\left\|f\left(s, \bar{z}_{k, s}+y_{s}, \int_{0}^{s} q\left(s, \tau, \bar{z}_{k, \tau}+y_{\tau}\right) d \tau\right)\right\| d s
\end{aligned}
$$


Since

$$
\begin{aligned}
& \left\|\int_{0}^{t} U(t, s) A(s) g\left(s, \bar{z}_{k, s}+y_{s}, \int_{0}^{s} h\left(s, \tau, \bar{z}_{k, \tau}+y_{\tau}\right) d \tau\right) d s\right\| \\
& \leq \int_{0}^{t}\|U(t, s)\|\left\|A(s) g\left(s, \bar{z}_{k, s}+y_{s}, \int_{0}^{s} h\left(s, \tau, \bar{z}_{k, \tau}+y_{\tau}\right) d \tau\right)\right\| d s \\
& \leq a M_{1} L_{1}\left(1+a M_{2} L_{3}\right)\left(k K_{a}+N+1\right), \\
& \left\|g\left(t, \bar{z}_{k, t}+y_{t}, \int_{0}^{t} h\left(t, s, \bar{z}_{k, s}+y_{s}\right) d s\right)\right\| \\
& =\left\|A^{-1}(t) A(t) g\left(t, \bar{z}_{k, t}+y_{t}, \int_{0}^{t} h\left(t, s, \bar{z}_{k, s}+y_{s}\right) d s\right)\right\| \\
& \leq M_{2} L_{1}\left(1+a M_{2} L_{3}\right)\left(k K_{a}+N+1\right), \\
& \int_{0}^{t}\left\|f\left(s, \bar{z}_{k, s}+y_{s}, \int_{0}^{s} q\left(s, \tau, \bar{z}_{k, \tau}+y_{\tau}\right) d \tau\right)\right\| d s \leq \int_{0}^{a} \mu_{k^{*}}(s) d s,
\end{aligned}
$$

where $k^{*}=(1+\delta)\left(k K_{a}+N\right)$, there holds

$$
\begin{gathered}
k<M_{1}\|g(0, \phi, 0)\|+M_{2} L_{1}\left(1+a M_{2} L_{3}\right)\left(k K_{a}+N+1\right)+a M_{1} L_{1}\left(1+a M_{2} L_{3}\right)\left(k K_{a}+N+1\right) \\
+a M_{1} M_{3}\left\{M_{1}[\|\phi(0)\|+\|g(0, \phi, 0)\|]+M_{2} L_{1}\left(1+a M_{2} L_{3}\right)\left(k K_{a}+N+1\right)\right. \\
\left.\quad+a M_{1} L_{1}\left(1+a M_{2} L_{3}\right)\left(k K_{a}+N+1\right)+M_{1} \int_{0}^{a} \mu_{k^{*}}(\tau) d \tau\right\}+M_{1} \int_{0}^{t} \mu_{k^{*}}(s) d s \\
=M^{*}+\left(1+a M_{1} M_{3}\right)\left(M_{2} L_{1} k K_{a}+a M_{2}^{2} L_{1} L_{3} k K_{a}\right) \\
+\left(1+a M_{1} M_{3}\right)\left(a M_{1} L_{1} k K_{a}+a^{2} M_{1} M_{2} L_{1} L_{3} k K_{a}\right)+\left(1+a M_{1} M_{3}\right) M_{1} \int_{0}^{a} \mu_{k^{*}}(s) d s \\
=M^{*}+\left(1+a M_{1} M_{3}\right)\left[M_{2} L_{1} k K_{a}+a M_{2}^{2} L_{1} L_{3} k K_{a}+a M_{1} L_{1} k K_{a}+a^{2} M_{1} M_{2} L_{1} L_{3} k K_{a}\right. \\
\left.\quad+(1+\delta)\left(k K_{a}+N\right) M_{1}\left[(1+\delta)\left(k K_{a}+N\right)\right]^{-1} \int_{0}^{a} \mu_{k^{*}}(s) d s\right] .
\end{gathered}
$$

Dividing both sides by $k$ and taking the lower limit, we get

$$
\left(1+a M_{1} M_{3}\right)\left[\left(M_{2} L_{1} K_{a}+a M_{1} L_{1} K_{a}\right)\left(1+a M_{2} L_{3}\right)+M K_{a}(1+\delta) \gamma\right] \geq 1
$$

This contradicts (2.14). Hence $P B_{k} \subseteq B_{k}$, for some positive number $k$. 
Now define the operators $P_{1}, P_{2}$ on $B_{k}$ by

$$
\begin{aligned}
\left(P_{1} z\right)(t)= & -U(t, 0) g(0, \phi, 0)+g\left(t, \bar{z}_{t}+y_{t}, \int_{0}^{t} h\left(t, s, \bar{z}_{s}+y_{s}\right) d s\right) \\
& +\int_{0}^{t} U(t, s) A(s) g\left(s, \bar{z}_{s}+y_{s}, \int_{0}^{s} h\left(s, \tau, \bar{z}_{\tau}+y_{\tau}\right) d \tau\right) d s, \\
\left(P_{2} z\right)(t)= & \int_{0}^{t} U(t, s) B u(s) d s+\int_{0}^{t} U(t, s) f\left(s, \bar{z}_{s}+y_{s}, \int_{0}^{s} q\left(s, \tau, \overline{z_{\tau}}+y_{\tau}\right) d \tau\right) d s
\end{aligned}
$$

for $0 \leq t \leq a$, respectively. We will show that $P_{1}$ is a contraction mapping and $P_{2}$ is a compact operator.

To prove that $P_{1}$ is a contraction, we take $z_{1}, z_{2} \in B_{k}$, then for each $t \in[0, a]$ and by (A1)(iii) and (2.6), (2.13), we have

$$
\begin{aligned}
& \left\|\left(P_{1} z_{1}\right)(t)-\left(P_{1} z_{2}\right)(t)\right\| \\
& \leq\left\|g\left(t, \bar{z}_{1, t}+y_{t}, \int_{0}^{t} h\left(t, s, \bar{z}_{1, s}+y_{s}\right) d s\right)-g\left(t, \bar{z}_{2, t}+y_{t}, \int_{0}^{t} h\left(t, s, \bar{z}_{2, s}+y_{s}\right) d s\right)\right\| \\
& +\| \int_{0}^{t} U(t, s) A(s)\left[g\left(s, \bar{z}_{1, s}+y_{s}, \int_{0}^{s} h\left(s, \tau, \bar{z}_{1, \tau}+y_{\tau}\right) d \tau\right)\right. \\
& \left.\quad-g\left(s, \bar{z}_{2, s}+y_{s}, \int_{0}^{s} h\left(s, \tau, \bar{z}_{2, \tau}+y_{\tau}\right) d \tau\right)\right] d s \| \\
& \leq M_{2} L\left(\left\|\bar{z}_{1, t}-\bar{z}_{2, t}\right\|_{\mathscr{B}}+\left\|\int_{0}^{t} h\left(t, s, \bar{z}_{1, s}+y_{s}\right) d s-\int_{0}^{t} h\left(t, s, \bar{z}_{2, s}+y_{s}\right) d s\right\|_{X}\right) \\
& +\int_{0}^{t} M_{1} L\left(\left\|\bar{z}_{1, s}-\bar{z}_{2, s}\right\|_{\mathscr{B}}+\left\|\int_{0}^{s} h\left(s, \tau, \bar{z}_{1, \tau}+y_{\tau}\right) d \tau-\int_{0}^{s} h\left(s, \tau, \bar{z}_{2, \tau}+y_{\tau}\right) d \tau\right\|_{X}\right) d s \\
& \leq\left(M_{2} L K_{a}+a M_{1} L K_{a}\right)\left(1+a M_{2} L_{2}\right) \sup _{0 \leq s \leq a}\left\|z_{1}(s)-z_{2}(s)\right\|=L^{*} \sup _{0 \leq s \leq a}\left\|z_{1}(s)-z_{2}(s)\right\| .
\end{aligned}
$$

Thus

$$
\left\|P_{1} z_{1}-P_{1} z_{2}\right\| \leq L^{*}\left\|z_{1}-z_{2}\right\|
$$

and so $P_{1}$ satisfies contraction condition with $L^{*}<1$.

To prove that $P_{2}$ is compact, first we prove that $P_{2}$ is continuous on $B_{k}$. Let $\left\{z_{n}\right\} \subseteq B_{k}$ with $z_{n} \rightarrow z$ in $B_{k}$, then for each $s \in[0, a], \bar{z}_{n, s} \rightarrow \bar{z}_{s}$ and by (H4)(i), we have

$$
f\left(s, \bar{z}_{n, s}+y_{s}, \int_{0}^{s} q\left(s, \tau, \bar{z}_{n, \tau}+y_{\tau}\right) d \tau\right) \longrightarrow f\left(s, \bar{z}_{s}+y_{s}, \int_{0}^{s} q\left(s, \tau, \overline{z_{\tau}}+y_{\tau}\right) d \tau\right) \quad \text { as } n \longrightarrow \infty \text {. }
$$


Since

$$
\left\|f\left(s, \bar{z}_{n, s}+y_{s}, \int_{0}^{s} q\left(s, \tau, \bar{z}_{n, \tau}+y_{\tau}\right) d \tau\right)-f\left(s, \bar{z}_{s}+y_{s}, \int_{0}^{s} q\left(s, \tau, \overline{z_{\tau}}+y_{\tau}\right) d \tau\right)\right\| \leq 2 \mu_{k^{*}}(s),
$$

then by the condition ( $\mathrm{H} 1)$ and the dominated convergence theorem, we have

$$
\begin{aligned}
\left\|P_{2} z_{n}-P_{2} z\right\|=\sup _{0 \leq t \leq a} \| & \mid \int_{0}^{t} U(t, s) B\left[u_{n}(s)-u(s)\right] d s \\
& +\int_{0}^{t} U(t, s)\left[f\left(s, \bar{z}_{n, s}+y_{s}, \int_{0}^{s} q\left(s, \tau, \bar{z}_{n, \tau}+y_{\tau}\right) d \tau\right)\right. \\
& \left.-f\left(s, \bar{z}_{s}+y_{s}, \int_{0}^{s} q\left(s, \tau, \bar{z}_{\tau}+y_{\tau}\right) d \tau\right)\right] d s \| \longrightarrow \text { as } n \rightarrow \infty,
\end{aligned}
$$

that is, $P_{2}$ is continuous.

Next we prove that the family $\left\{P_{2} z: z \in B_{k}\right\}$ is an equicontinuous family of functions. To do this, let $\epsilon>0$ be small, $0<t_{1}<t_{2}$, then

$$
\begin{aligned}
\|\left(P_{2} z\right)( & \left.t_{1}\right)-\left(P_{2} z\right)\left(t_{2}\right) \| \\
= & \| \int_{0}^{t_{1}} U\left(t_{1}, s\right) B u(s) d s+\int_{0}^{t_{1}} U\left(t_{1}, s\right) f\left(s, \bar{z}_{s}+y_{s}, \int_{0}^{s} q\left(s, \tau, \overline{z_{\tau}}+y_{\tau}\right) d \tau\right) d s \\
& -\int_{0}^{t_{2}} U\left(t_{2}, s\right) B u(s) d s-\int_{0}^{t_{2}} U\left(t_{2}, s\right) f\left(s, \bar{z}_{s}+y_{s}, \int_{0}^{s} q\left(s, \tau, \overline{z_{\tau}}+y_{\tau}\right) d \tau\right) d s \| \\
\leq & \int_{0}^{t_{1}-\epsilon}\left\|U\left(t_{1}, s\right)-U\left(t_{2}, s\right)\right\|\|B\|\|u(s)\| d s \\
& +\int_{t_{1}-\epsilon}^{t_{1}}\left\|U\left(t_{1}, s\right)-U\left(t_{2}, s\right)\right\|\|B\|\|u(s)\| d s+\int_{t_{1}}^{t_{2}}\left\|U\left(t_{2}, s\right)\right\|\|B\|\|u(s)\| d s \\
& +\int_{0}^{t_{1}-\epsilon}\left\|U\left(t_{1}, s\right)-U\left(t_{2}, s\right)\right\|\left\|f\left(s, \bar{z}_{s}+y_{s}, \int_{0}^{s} q\left(s, \tau, \overline{z_{\tau}}+y_{\tau}\right) d \tau\right)\right\| d s \\
& +\int_{t_{1}-\epsilon}^{t_{1}}\left\|U\left(t_{1}, s\right)-U\left(t_{2}, s\right)\right\|\left\|f\left(s, \overline{z_{s}}+y_{s}, \int_{0}^{s} q\left(s, \tau, \overline{z_{\tau}}+y_{\tau}\right) d \tau\right)\right\| d s \\
& +\int_{t_{1}}^{t_{2}}\left\|U\left(t_{2}, s\right)\right\|\left\|f\left(s, \overline{z_{s}}+y_{s}, \int_{0}^{s} q\left(s, \tau, \overline{z_{\tau}}+y_{\tau}\right) d \tau\right)\right\| d s .
\end{aligned}
$$


Observe that

$$
\begin{aligned}
\|u(s)\| \leq\left\|\widetilde{W}^{-1}\right\| & \left\{M_{1}[\|\phi(0)\|+\|g(0, \phi, 0)\|]\right. \\
& +\left\|A^{-1}(a)\right\|\left\|A(a) g\left(a, \bar{z}_{a}+y_{a}, \int_{0}^{a} h\left(a, s, \overline{z_{s}}+y_{s}\right) d s\right)\right\| \\
& +\int_{0}^{a}\|U(a, \tau)\|\left\|A(\tau) g\left(\tau, \overline{z_{\tau}}+y_{\tau}, \int_{0}^{\tau} h\left(\tau, \eta, \overline{z_{\eta}}+y_{\eta}\right) d \eta\right)\right\| d \tau \\
& \left.+\int_{0}^{a}\|U(a, \tau)\|\left\|f\left(\tau, \bar{z}_{\tau}+y_{\tau}, \int_{0}^{\tau} q\left(\tau, \eta, \overline{z_{\eta}}+y_{\eta}\right) d \eta\right)\right\| d \tau\right\} \\
\leq\|\widetilde{W}-1\|\{ & M_{1}[\|\phi(0)\|+\|g(0, \phi, 0)\|]+M_{2} L_{1}\left(1+a M_{2} L_{3}\right)\left(k K_{a}+N+1\right) \\
& \left.+a M_{1} L_{1}\left(1+a M_{2} L_{3}\right)\left(k K_{a}+N+1\right)+M_{1} \int_{0}^{a} \mu_{k^{*}}(\tau) d \tau\right\}
\end{aligned}
$$

and $\mu_{k^{*}}(\tau) \in L^{1}$, we see that $\left\|\left(P_{2} z\right)\left(t_{1}\right)-\left(P_{2} z\right)\left(t_{2}\right)\right\|$ tends to zero independent of $z \in B_{k}$ as $\left(t_{2}-t_{1}\right) \rightarrow 0$ with $\epsilon$ sufficiently small, since the compactness of $U(t, s)$ implies the continuity of $U(t, s)$ in the uniform operator topology. Hence, $P_{2}$ maps $B_{k}$ into an equicontinuous family of functions.

It remains to prove that $V(t)=\left\{\left(P_{2} z\right)(t): z \in B_{k}\right\}$ is relatively compact in $X$. Let $0<$ $t \leq a$ be fixed, $0<\epsilon<t$. For $z \in B_{k}$, we define

$$
\begin{aligned}
\left(P_{2, \epsilon} z\right)(t) & =\int_{0}^{t-\epsilon} U(t, s)\left[B u(s)+f\left(s, \bar{z}_{s}+y_{s}, \int_{0}^{s} q\left(s, \tau, \overline{z_{\tau}}+y_{\tau}\right) d \tau\right)\right] d s \\
& =U(t, t-\epsilon) \int_{0}^{t-\epsilon} U(t-\epsilon, s)\left[B u(s)+f\left(s, \bar{z}_{s}+y_{s}, \int_{0}^{s} q\left(s, \tau, \bar{z}_{\tau}+y_{\tau}\right) d \tau\right)\right] d s .
\end{aligned}
$$

Using the estimation of $\|u(s)\|$ and by the compactness of $U(t, s)$ we prove that $V_{\epsilon}(t)=$ $\left\{\left(P_{2, \epsilon} z\right)(t): z \in B_{k}\right\}$ is relatively compact in $X$ for every $\epsilon, 0<\epsilon<t$. Moreover, for every $z \in B_{k}$, we have

$$
\begin{aligned}
& \left\|\left(P_{2} z\right)(t)-\left(P_{2, \epsilon} z\right)(t)\right\| \\
& \leq \int_{t-\epsilon}^{t}\left\|U(t, s)\left[B u(s)+f\left(s, \bar{z}_{s}+y_{s}, \int_{0}^{s} q\left(s, \tau, \overline{z_{\tau}}+y_{\tau}\right) d \tau\right)\right]\right\| d s \\
& \leq \int_{t-\epsilon}^{t} M_{1}\left\{M _ { 3 } \left[M_{1}[\|\phi(0)\|+\|g(0, \phi, 0)\|]+M_{2} L_{1}\left(1+a M_{2} L_{3}\right)\left(k K_{a}+N+1\right)\right.\right. \\
& \left.\left.\quad+a M_{1} L_{1}\left(1+a M_{2} L_{3}\right)\left(k K_{a}+N+1\right)+M_{1} \int_{0}^{a} \mu_{k^{*}}(\tau) d \tau\right]+\mu_{k^{*}}(s)\right\} d s .
\end{aligned}
$$

Therefore there are relatively compact sets arbitrarily close to the set $V(t)=\left\{\left(P_{2} z\right)(t)\right.$ : $\left.z \in B_{k}\right\}$, hence the set $V(t)$ is also relatively compact in $X$. 
Thus by the Arzela-Ascoli theorem $P_{2}$ is a compact operator. These arguments show that $P=P_{1}+P_{2}$ is a condensing mapping on $B_{k}$, and by the Sadovskiu fixed point theorem [17] there exists a fixed point $z(\cdot)$ for $P$ on $B_{k}$. If we define $x(t)=z(t)+y(t),-\infty<t \leq a$, then it is easy to see that $x(\cdot)$ is a mild solution of (1.8) satisfying $x_{0}=\phi, x(a)=0$. Hence the proof.

Now we discuss the local null controllability of the system (1.8). For this purpose, we impose the weaker assumptions on the system (1.8) as follows.

(H7) $g: J \times \Omega \times X \rightarrow X$ is a continuous function, and there exists a constant $L>0$ such that the function $A(t) g$ satisfies the Lipschitz condition:

$$
\begin{aligned}
& \left\|A(t) g\left(s_{1}, \phi_{1}, \eta_{1}\right)-A(t) g\left(s_{2}, \phi_{2}, \eta_{2}\right)\right\| \\
& \quad \leq L\left[\left|s_{1}-s_{2}\right|+\left\|\phi_{1}-\phi_{2}\right\|_{\Re}+\left\|\eta_{1}-\eta_{2}\right\|_{X}\right] \quad \text { for } 0 \leq s_{1}, s_{2} \leq a .
\end{aligned}
$$

(H8) Let $\Omega \subset \mathscr{B}$ be an open set and let the function $f: J \times \Omega \times X \rightarrow X$ satisfy the following conditions.

(i) For each $t \in[0, a]$, the function $f(t, \cdot, \cdot): \Omega \times X \rightarrow X$ is continuous and for each $(\phi, x) \in \Omega \times X$ the function $f(\cdot, \phi, x): J \rightarrow X$ is strongly measurable.

(ii) For each positive number $k$, there is a positive function $\mu_{k} \in L^{1}([0, a])$ such that

$$
\sup _{\|x\|,\|y\| \leq k}\|f(t, x, y)\| \leq \mu_{k}(t)
$$

Theorem 3.2. If the assumptions (H5), (H7), and (H8) are satisfied and $\phi \in \Omega$, then the system (1.8) is locally controllable on the interval $[0, a]$.

Proof. We prove this theorem by using again the Sadovskil fixed point theorem. Let $y(\cdot)$, $u(\cdot), S, P, P_{1}, P_{2}$ be as in the proof of Theorem 3.1. It is enough to prove that $P$ has a fixed point, which implies that $S$ has a fixed point.

Since $A(t) g(\cdot, \cdot)$ satisfies Lipschitz condition and $\Omega$ is open, there exist $0<b_{1}<b$, $r>0$, such that $B_{r}(\phi) \in \Omega$ and

$$
\|A(t) g(t, \psi, \eta)\| \leq C_{1}
$$

for some constant $C_{1} \geq 0$ and $(t, \psi, \eta) \in\left[0, b_{1}\right] \times B_{r}(\phi) \times X$. As $y_{0}=\phi$, we choose $0<b_{2}<$ $b_{1}$ such that $\left\|y_{t}-\phi\right\|_{\Re} \leq r / 2$ for all $0 \leq t \leq b_{2}$.

Let $\rho=r / 2 K_{b_{2}}$ and $\epsilon>0$, then from the continuity of the functions $g, K(t)$ and $t \rightarrow y_{t}$, the compactness of the set $\left\{g\left(t, y_{t}, \int_{0}^{t} h\left(t, s, y_{s}\right) d s\right): 0 \leq t \leq b_{2}\right\}$, and the absolute continuity of Lebesgue integral, it follows that there exists a constant $a, 0<a<b_{2}$, for which the 
14 Null controllability of neutral integrodifferential systems

following hold:

$$
\begin{gathered}
\left\|y_{t}-\phi\right\|_{\mathscr{B}} \leq \epsilon, \\
\left\|(U(t, 0)+I) g\left(t, y_{t}, \int_{0}^{t} h\left(t, s, y_{s}\right) d s\right)\right\| \leq \epsilon \\
\left(a M_{1} M_{3}+1\right)\left(M_{1} M_{2} L\left(a+\epsilon+a M_{2} L_{3} N+a M_{2} L_{3}\right)+\epsilon+M_{1} C_{1} a\right) \\
\leq \frac{1}{3}\left[1-\left(a M_{1} M_{3}+1\right) M_{2} L K_{a}\left(1+a M_{2} L_{2}\right)\right] \rho \\
\left(a M_{1} M_{3}+1\right) M_{1} \int_{0}^{a} \mu_{k^{*}}(s) d s \leq \frac{1}{3}\left[1-\left(a M_{1} M_{3}+1\right) M_{2} L K_{a}\left(1+a M_{2} L_{2}\right)\right] \rho, \\
a M_{1} M_{3}\left(M_{1}\|\phi(0)\|\right) \leq \frac{1}{3}\left[1-\left(a M_{1} M_{3}+1\right) M_{2} L K_{a}\left(1+a M_{2} L_{2}\right)\right] \rho, \\
\left(M_{2} L K_{a}+a M_{1} L K_{a}\right)\left(1+a M_{2} L_{2}\right)<1
\end{gathered}
$$

for all $0 \leq t \leq a$.

Define the set

$$
S(\rho)=\{z \in C([0, a] ; X): z(0)=0,\|z(t)\| \leq \rho, 0 \leq t \leq a\},
$$

then $S(\rho)$ is also a nonempty bounded, closed, and convex subset of $C([0, a] ; X)$ and $P$ is well defined on $S(\rho)$. We will show that $P$ maps $S(\rho)$ into $S(\rho)$. In fact, let $z \in S(\rho)$; we have

$$
\begin{aligned}
\left(P_{1} z\right)(t)= & -U(t, 0) g(0, \phi, 0)+g\left(t, \bar{z}_{t}+y_{t}, \int_{0}^{t} h\left(t, s, \bar{z}_{s}+y_{s}\right) d s\right) \\
& +\int_{0}^{t} U(t, s) A(s) g\left(s, \bar{z}_{s}+y_{s}, \int_{0}^{s} h\left(s, \tau, \bar{z}_{\tau}+y_{\tau}\right) d \tau\right) d s \\
= & U(t, 0) A^{-1}(t)\left[-A(t) g(0, \phi, 0)-A(t) g\left(t, y_{t}, \int_{0}^{t} h\left(t, s, y_{s}\right) d s\right)\right] \\
& +(U(t, 0)+I) g\left(t, y_{t}, \int_{0}^{t} h\left(t, s, y_{s}\right) d s\right) \\
& -A^{-1}(t)\left[A(t) g\left(t, y_{t}, \int_{0}^{t} h\left(t, s, y_{s}\right) d s\right)-A(t) g\left(t, \bar{z}_{t}+y_{t}, \int_{0}^{t} h\left(t, s, \bar{z}_{s}+y_{s}\right) d s\right)\right] \\
& +\int_{0}^{t} U(t, s) A(s) g\left(s, \bar{z}_{s}+y_{s}, \int_{0}^{s} h\left(s, \tau, \bar{z}_{\tau}+y_{\tau}\right) d \tau\right) d s,
\end{aligned}
$$


then from condition (H7) and (3.27) and (3.28), it yields

$$
\begin{aligned}
\left\|\left(P_{1} z\right)(t)\right\| \leq & \|U(t, 0)\|\left\|A^{-1}(t)\right\| L\left[t+\left\|y_{t}-\phi\right\|_{\Re}+\left\|\int_{0}^{t} h\left(t, s, y_{s}\right) d s\right\|_{X}\right] \\
& +\left\|(U(t, 0)+I) g\left(t, y_{t}, \int_{0}^{t} h\left(t, s, y_{s}\right) d s\right)\right\| \\
& +\left\|A^{-1}(t)\right\| L\left[\left\|\bar{z}_{t}\right\|_{\Re}+\left\|\int_{0}^{t} h\left(t, s, \bar{z}_{s}+y_{s}\right) d s-\int_{0}^{t} h\left(t, s, y_{s}\right) d s\right\|_{X}\right] \\
& +M_{1} C_{1} t \leq M_{1} M_{2} L\left[a+\epsilon+a M_{2} L_{3} N+a M_{2} L_{3}\right]+\epsilon+M_{1} C_{1} a \\
& +M_{2} L \rho K_{a}\left(1+a M_{2} L_{2}\right) .
\end{aligned}
$$

Thus by using (3.29)-(3.31) we derive that

$$
\begin{aligned}
& \left\|\left(P z_{k}\right)(t)\right\| \\
& =\|-U(t, 0) g(0, \phi, 0)+g\left(t, \bar{z}_{k, t}+y_{t}, \int_{0}^{t} h\left(t, s, \bar{z}_{k, s}+y_{s}\right) d s\right) \\
& +\int_{0}^{t} U(t, s) A(s) g\left(s, \bar{z}_{k, s}+y_{s}, \int_{0}^{s} h\left(s, \tau, \bar{z}_{k, \tau}+y_{\tau}\right) d \tau\right) d s \\
& +\int_{0}^{t} U(t, s) B \widetilde{W}^{-1}\{-U(a, 0)[\phi(0)-g(0, \phi, 0)] \\
& -g\left(a, \bar{z}_{k, a}+y_{a}, \int_{0}^{a} h\left(a, s, \bar{z}_{k, s}+y_{s}\right) d s\right) \\
& -\int_{0}^{a} U(a, s) A(s) g\left(s, \bar{z}_{k, s}+y_{s}, \int_{0}^{s} h\left(s, \tau, \bar{z}_{k, \tau}+y_{\tau}\right) d \tau\right) d s \\
& \left.-\int_{0}^{a} U(a, s) f\left(s, \bar{z}_{k, s}+y_{s}, \int_{0}^{s} q\left(s, \tau, \bar{z}_{k, \tau}+y_{\tau}\right) d \tau\right) d s\right\}(s) d s \\
& +\int_{0}^{t} U(t, s) f\left(s, \bar{z}_{k, s}+y_{s}, \int_{0}^{s} q\left(s, \tau, \bar{z}_{k, \tau}+y_{\tau}\right) d \tau\right) d s \| \\
& \leq\left(a M_{1} M_{3}+1\right)\left(M_{1} M_{2} L\left(a+\epsilon+a M_{2} L_{3} N+a M_{2} L_{3}\right)\right. \\
& \left.+\epsilon+M_{1} C_{1} a+M_{2} L \rho K_{a}\left(1+a M_{2} L_{2}\right)\right) \\
& +\left(a M_{1} M_{3}+1\right) M_{1} \int_{0}^{a} \mu_{k^{*}}(s) d s+a M_{1} M_{3}\left(M_{1}\|\phi(0)\|\right) \\
& \leq 3 \times \frac{1}{3}\left[1-\left(a M_{1} M_{3}+1\right) M_{2} L K_{a}\left(1+a M_{2} L_{2}\right)\right] \rho \\
& +\left(a M_{1} M_{3}+1\right)\left(M_{2} L \rho K_{a}\left(1+a M_{2} L_{2}\right)\right) \leq \rho .
\end{aligned}
$$

The remaining part of the proof is similar to that of Theorem 3.1 and hence it is omitted. 


\section{Example}

Consider the following partial functional differential system:

$$
\begin{gathered}
\frac{\partial}{\partial t}\left[z(t, x)+\int_{0}^{\pi} b(y, x) z(t \sin t, y) d y\right] \\
=a(t, x) \frac{\partial^{2}}{\partial x^{2}} z(t, x)+\mu(t, x)+\int_{0}^{t} h\left(t, s, z_{s}\right) d s, \quad 0 \leq t \leq a, 0 \leq x \leq \pi, \\
z(t, 0)=z(t, \pi)=0, \\
z(0, x)=z_{0}(x), \quad 0 \leq x \leq \pi,
\end{gathered}
$$

where $a(t, x)$ is a continuous function and is uniformly Holder continuous in $t$. Let $X=$ $L^{2}([0, \pi])$ and let $A(t)$ be defined by

$$
A(t) z=-a(t, x) z^{\prime \prime}
$$

with the domain

$$
D(A)=\left\{z(\cdot) \in X: z, z^{\prime} \text { absolutely continuous, } z^{\prime \prime} \in X, z(0)=z(\pi)=0\right\} .
$$

Then $A(t)$ generates an evolution system $U(t, s)$ satisfying assumptions (B1)-(B4).

Here we take the phase space $\mathscr{B}=C_{0} \times L^{2}(g ; X)$, which contains all classes of functions $\phi:(-\infty, 0] \rightarrow X$ such that $\phi$ is Lebesgue measurable and $g(\cdot)\|\phi(\cdot)\|^{2}$ is Lebesgue integrable on $(-\infty, 0)$ where $g:(-\infty, 0) \rightarrow R$ is a positive integrable function. The seminorm in $\mathscr{B}$ is defined by

$$
\|\phi\|_{\mathscr{B}}=\|\phi(0)\|+\left(\int_{-\infty}^{0} g(\theta)\|\phi(\theta)\|^{2} d \theta\right)^{1 / 2} .
$$

Assume the following conditions hold.

(i) The function $b$ is measurable and

$$
\iint_{0}^{\pi} b^{2}(y, x) d y d x<\infty
$$

(ii) The function $(\partial / \partial x) b(y, x)$ is measurable, $b(y, 0)=b(y, \pi)=0$, and let

$$
N_{1}=\left[\iint_{0}^{\pi}\left(\frac{\partial}{\partial x} b(y, x)\right)^{2} d y d x\right]^{1 / 2}<\infty .
$$

(iii) For the function $h:[0, a] \times[0, a] \times \mathscr{B} \rightarrow X$ the following conditions are satisfied. (iii.a) For each $t, s \in[0, a], h(t, s, \cdot)$ is continuous.

(iii.b) For each $z \in X, h\left(\cdot, \cdot, z_{s}\right)$ is measurable.

(iii.c) There are positive functions $h_{1}, h_{2} \in L^{1}([0, a])$ such that

$$
|h(t, s, \phi)| \leq h_{1}(t)|\phi|+h_{2}(s)
$$

for all $(t, s, \phi) \in[0, a] \times[0, a] \times \mathscr{B}$. 
(iv) The function $u: J \rightarrow U \subset X$ is defined by

$$
(u(t))(x)=\mu(x, t), \quad x \in[0, \pi],
$$

where $\mu:[0, \pi] \times J \rightarrow[0, \pi]$ is continuous.

(v) The linear operator $W$ from $L^{2}(J ; U)$ into $X$ is defined by

$$
W u=\int_{0}^{a} U(a, s) B u(s) d s
$$

which induces an invertible operator $\widetilde{W}$ defined on $L^{2}(J ; U) / \operatorname{ker} W$.

We define $f, g: J \times \mathscr{B} \times X \rightarrow X$ by $g\left(t, z_{t}, z\right)=Z_{1}(z), f\left(t, z_{t}, z\right)(x)=\int_{0}^{t} h\left(t, s, z_{s}\right) d s$, respectively, where

$$
Z_{1}(z)(x)=\int_{0}^{\pi} b(y, x) z(y) d y
$$

From (i) it is clear that $Z_{1}$ is a bounded linear operator on $\mathscr{B}$. Furthermore, by (4.2) and (iii) $Z_{1}(z) \in D(A)$ and $\left\|A(t) Z_{1}(z)\right\| \leq N_{1}$. In fact, from the definition of $Z_{1}$ and (ii) it follows that

$$
\left\langle Z_{1}(z), z_{n}\right\rangle=\int_{0}^{\pi} z_{n}(x)\left[\int_{0}^{\pi} b(y, x) z(y) d y\right] d x=\frac{1}{n}\left(\frac{2}{\pi}\right)^{1 / 2}\langle Z(z), \cos (n x)\rangle,
$$

where $Z$ is defined by

$$
Z(z)(x)=\int_{0}^{\pi} \frac{\partial}{\partial x} b(y, x) z(y) d y .
$$

From (ii) we know that $Z: X \rightarrow X$ is a bounded linear operator with $\|Z\| \leq N_{1}$. Hence $\left\|A(t) Z_{1}(z)\right\|=\|Z(z)\|$ verifies the assumption (H1). Assumptions (H2) and (H3) are obviously satisfied. Hence from Theorem 3.1, the system (4.1) is null controllable on $[0, a]$ under the above assumptions provided that (2.13) and (2.14) hold.

\section{References}

[1] I. M. Ananjevskiı̆ and V. B. Kolmanovskiŭ, Stabilization of some nonlinear hereditary mechanical systems, Nonlinear Analysis. Theory, Methods \& Applications 15 (1990), no. 2, 101-114.

[2] K. Balachandran and E. R. Anandhi, Neutral functional integrodifferential control systems in $\mathrm{Ba}$ nach spaces, Kybernetika 39 (2003), no. 3, 359-367.

[3] K. Balachandran and J. P. Dauer, Existence of solutions of a nonlinear mixed neutral equation, Applied Mathematics Letters 11 (1998), no. 2, 23-28.

[4] Controllability of nonlinear systems in Banach spaces: a survey, Journal of Optimization Theory and Applications 115 (2002), no. 1, 7-28.

[5] E. N. Chukwu, Differential Models and Neutral Systems for Controlling the Wealth of Nations, Series on Advances in Mathematics for Applied Sciences, vol. 54, World Scientific, New Jersey, 2001.

[6] C. Corduneanu and V. Lakshmikantham, Equations with unbounded delay: a survey, Nonlinear Analysis. Theory, Methods \& Applications 4 (1980), no. 5, 831-877.

[7] A. Friedman, Partial Differential Equations, Holt, Rinehart and Winston, New York, 1969. 
[8] X. L. Fu, Controllability of neutral functional differential systems in abstract space, Applied Mathematics and Computation 141 (2003), no. 2-3, 281-296.

[9] Controllability of abstract neutral functional differential systems with unbounded delay, Applied Mathematics and Computation 151 (2004), no. 2, 299-314.

[10] X. L. Fu and K. Ezzinbi, Existence of solutions for neutral functional differential evolution equations with nonlocal conditions, Nonlinear Analysis 54 (2003), no. 2, 215-227.

[11] J. K. Hale and J. Kato, Phase space for retarded equations with infinite delay, Funkcialaj Ekvacioj 21 (1978), no. 1, 11-41.

[12] H. R. Henríquez, Periodic solutions of quasi-linear partial functional-differential equations with unbounded delay, Funkcialaj Ekvacioj 37 (1994), no. 2, 329-343.

[13] _ Regularity of solutions of abstract retarded functional-differential equations with unbounded delay, Nonlinear Analysis. Theory, Methods \& Applications 28 (1997), no. 3, 513-531.

[14] E. Hernández and H. R. Henríquez, Existence results for partial neutral functional-differential equations with unbounded delay, Journal of Mathematical Analysis and Applications 221 (1998), no. $2,452-475$.

[15] Y. Hino, S. Murakami, and T. Naito, Functional-Differential Equations with Infinite Delay, Lecture Notes in Mathematics, vol. 1473, Springer, Berlin, 1991.

[16] W. H. Kwon, G. W. Lee, and S. W. Kim, Performance improvement using time delays in multivariable controller design, International Journal of Control 52 (1990), no. 6, 1455-1473.

[17] B. N. Sadovskiŭ, On a fixed point principle, Functional Analysis and Applications 1 (1967), no. 2, 74-76.

[18] P. Sobolevskii, On equations of parabolic type in Banach space, American Mathematical Society Translations 49 (1965), 1-62.

K. Balachandran: Department of Mathematics, Bharathiar University, Coimbatore 641 046, India E-mail address: balachandran_k@lycos.com

A. Leelamani: Department of Mathematics, Bharathiar University, Coimbatore 641 046, India E-mail address: leelamani_a@rediffmail.com 


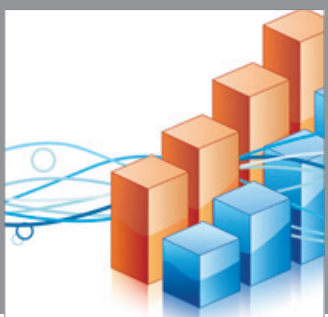

Advances in

Operations Research

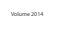

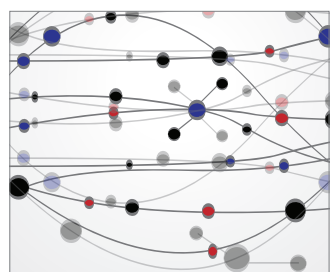

\section{The Scientific} World Journal
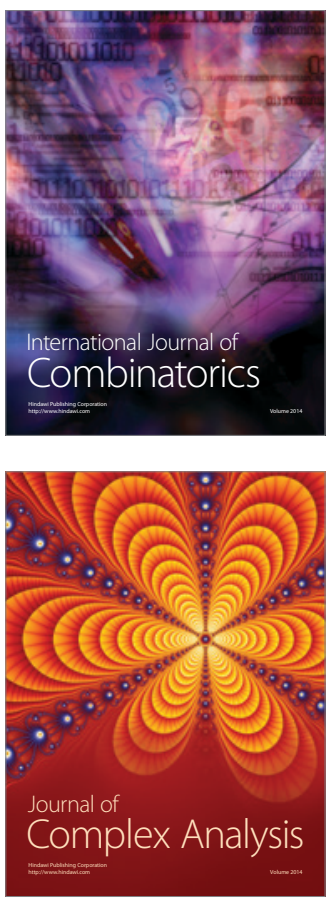

International Journal of

Mathematics and

Mathematical

Sciences
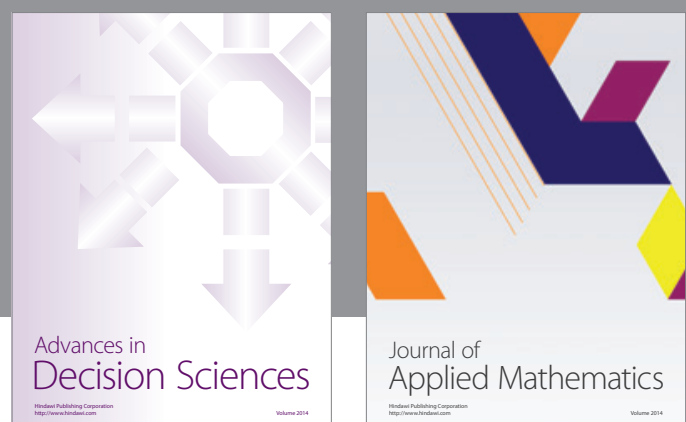

Journal of

Applied Mathematics
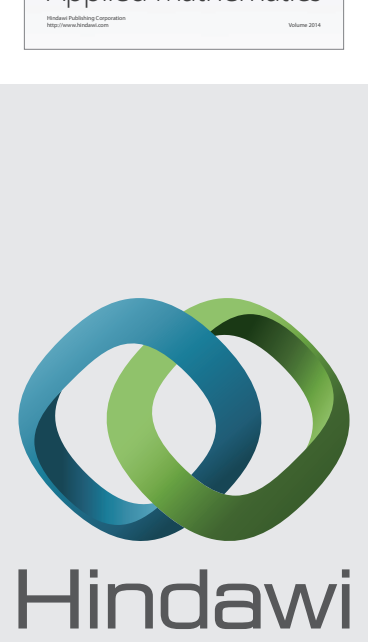

Submit your manuscripts at http://www.hindawi.com
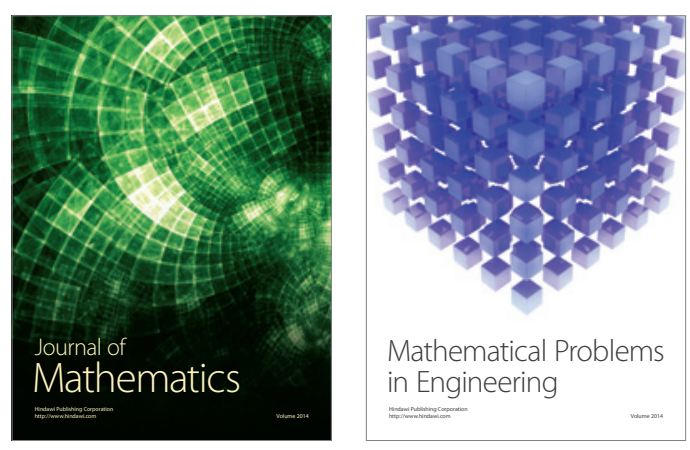

Mathematical Problems in Engineering
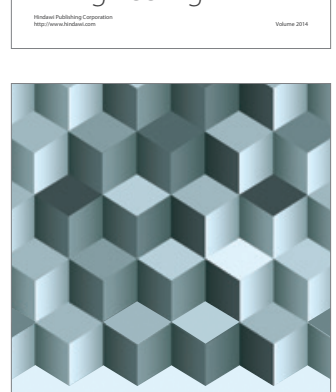

Journal of

Function Spaces
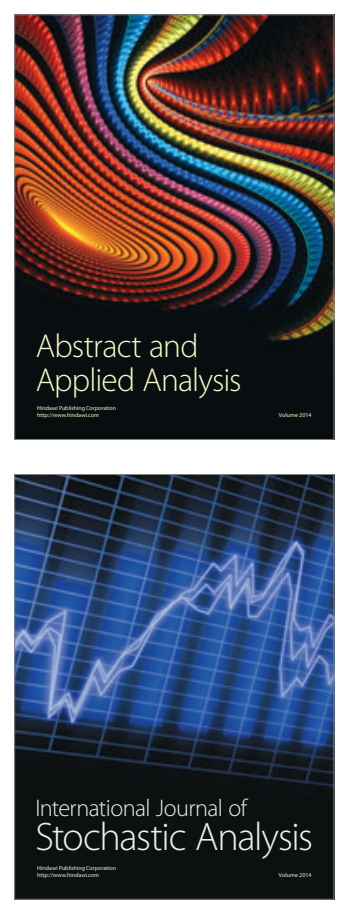

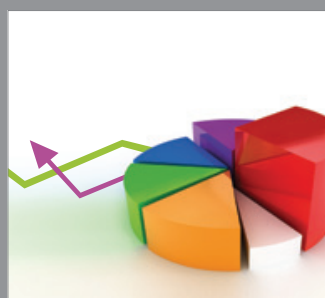

ournal of

Probability and Statistics

Promensencen
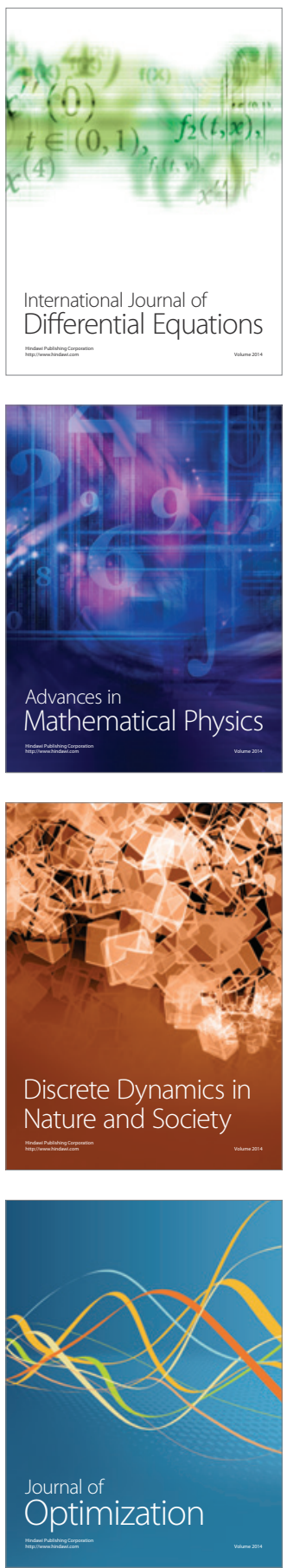